\title{
Thoracoscopic bidirectional S10 segmentectomy: a case report
}

\author{
Min Zhang, Guang Fu, Xu Chen, Ming-Jian Ge \\ Department of Cardiothoracic Surgery, the First Affiliated Hospital of Chongqing Medical University, Chongqing, China \\ Correspondence to: Ming-Jian Ge. Department of Cardiothoracic Surgery, the First Affiliated Hospital of Chongqing Medical University, Chongqing \\ 400016, China. Email: doctor1984@sina.com.
}

\begin{abstract}
Background: Segmentectomies of posterior basal segment (S10) is considered the most difficult anatomical segmentectomy. The S10 locates very deep inside the lung tissue, and far away from the oblique fissure. Besides, the cuboid-shaped intersegmental plane of S10 is hard to be fold into a two-dimensional plane with stapler.

Case Description: We describe a novel method of bidirectional S10 segmentectomy with single-blocking of pulmonary circulation. The purpose is to simplify the procedure of S10 segmentectomy. The advantages of this method are that it is easy to learn and use, without requirements of special and expensive instruments like indocyanine green (ICG) method. It could save time because the target bronchus is dissected and resected by utilizing the waiting period of intersegmental plane. Because the bronchus is not resected at the moment of inflation, it is easy to achieve a full re-expansion with a relatively low pressure. This could reduce pressure-related damage of lung caused by manual ventilation. The S10 segmentectomy is considered a difficult procedure among all segmentectomies.
\end{abstract}

Conclusion: Although different methods were introduced, this is the first time in literature that we combine the pulmonary circulation single-blocking method and "bidirectional" method together for S10 segmentectomy. The preliminary result shows that it might be a useful method for S10 segmentectomy. It is safe with acceptable complications.

Keywords: S10 segmentectomy; bidirectional; single-blocking; case report

Received: 12 July 2021; Accepted: 19 November 2021; Published: 30 January 2022.

doi: $10.21037 /$ shc-21-18

View this article at: https://dx.doi.org/10.21037/shc-21-18

\section{Introduction}

Segmentectomies of posterior basal segment (S10) is considered the most difficult anatomical segmentectomy. The S10 locates very deep inside the lung tissue, and far away from the oblique fissure. Besides, the cuboid-shaped intersegmental plane of S10 is hard to be fold into a twodimensional plane with stapler. We have been working on segmentectomy for long time, and several methods were introduced. One is the "bidirectional" method (1). According to this method, the anatomical identification is performed from the oblique fissure, and the target segment's resection is achieved from the posterior hilum. Another method is the "single-blocking" strategy of pulmonary circulation (2). It features by blocking firstly the artery or vein alone for the identification of the intersegmental plane, and then resecting the rest structures of target segment. Here we combine both of the methods together to simplify the procedure of S10 segmentectomy. Our selection criteria are as follows: patients with a $\leq 2$ $\mathrm{cm}$ ground glass opacity (GGO) dominant (GGO $\geq 50 \%$ ) peripheral pulmonary nodule that might be pre-invasive lung cancer, patients with multiple GGOs involving right middle lobe (RML), or patients with possible benign lesion. Patients who are not suitable for lobectomy because of compromised pulmonary function are also appropriate candidates for this parenchyma sparing procedure. Patients with GGO close to the visceral pleural which is suitable for a wedge resection is excluded. Patients with GGO locating in the inner one third of lung where a safe oncological margin cannot be guaranteed is also excluded. The surgical margin was designed to be more than $2 \mathrm{~cm}$ or larger than the maximum diameter of the tumor. The new proposed histologic classification system and the eighth edition 


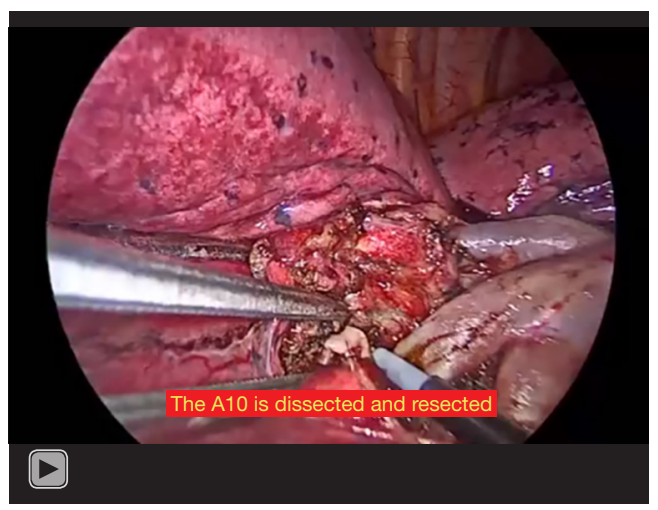

Video 1 Thoracoscopic bidirectional S10 segmentectomy.

of the TNM staging system were adopted for histologic typing and surgical-pathologic staging, respectively. We present the following article in accordance with the CARE reporting checklist (available at https://shc.amegroups. com/article/view/10.21037/shc-21-18/rc).

\section{Case presentation}

Traditional four ports are made to perform this surgery (1). The main utility incision and observing port was inserted in the fourth and seventh intercostal space in the anterior axillary line. Two assistant incisions were made in the seventh intercostal space in mid-axillary line and ninth intercostal space in posterior axillary line. The endostapler can be introduced in any of the port (except the observing port). Unlike the single-port VATS surgery, the angle of the endostaplers does not affect us to perform the surgery. With the help of the high-resolution computed tomography (HRCT), the positional relations of bronchus and vessels in target segmental were identified before surgery. The bidirectional S10 segmentectomy is started from the interlobar fissure. To achieve a correct orientation, an adequate dissection of pulmonary artery is required. It is usually easy to identify A6, A8 and A9. The target segment artery (A10) locates deep in the lung; it was dissected and resected. Then, we identify the target segmental bronchus (B10) by its running direction with artery stump. The B10 is dissected, but not transected at this moment. A sling is passed to encircle the B10. It is known that the factors influencing pulmonary circulation includes the artery and the vein. The blocking of either could result in an obstruction of pulmonary circulation. We therefore only resect the target artery of A10, and then inflate the lung. While waiting for the visualization of the intersegmental plane, the target bronchus of B10 is resected.

As the next step, the pulmonary ligament (PL) region is explored. This is why it is called "bidirectional". An adequate exposure of the branches of inferior pulmonary vein is necessary. Sato identifies the target vein branching preoperatively with the help of three-dimensional computed tomography bronchography and angiography (3D-CTBA) (3); however, we are different. we examine the target V10 according to the intersegmental plane: the vein which runs into the inflation area is V10. The veins which run into the deflation area are not V10 and need to be preserved. This could successfully avoid a major shortcoming of the PL approach: the manipulation during operation usually leads the surgeon to misunderstand the anatomy of vein, even with the help of 3D-CTBA. A stapler is used to tailor the intersegmental plane, which begins from the peripheral of the lung to the most difficult, hilar lung step by step (Video 1).

From July 2020 to January 2021, a total of 5 cases of S10 segmentectomy were performed. The average blood loss was $90 \pm 13 \mathrm{~mL}$. The average operative time was $118 \pm 23$ minutes. The average chest tube duration was $3 \pm 0.4$ days. The average hospital stay was $4 \pm 0.7$ days. There is no prolonged air leakage. No mortality was identified. Pathological examination revealed 2 cases of adenocarcinoma in situ (AIS) and 3 cases of minimally invasive adenocarcinoma (MIA). The average number of cartridges used is 5 . All procedures performed in studies involving human participants were in accordance with the ethical standards of the institutional and/or national research committee(s) and with the Helsinki Declaration (as revised in 2013). Written informed consent was obtained from the patients for publication of this case report and accompanying images. A copy of the written consent is available for review by the editorial office of this journal.

\section{Discussion}

The intersegmental plane's visualization plays a key role in segmentectomy. The modified inflation-deflation method is most commonly used (4). By making a small modification to the modified inflation-deflation method, we proposed the pulmonary circulation single-blocking method. In the traditional modified inflation-deflation method, at the moment when the intersegmental plane can be visualized, the target segment's bronchus and vessels are already resected; in other words, if there are mistakes, they are not correctable. Conversely, in the pulmonary circulation single-blocking method, at the moment when 
the intersegmental plane is visualized, not all the target segmental structures are resected. It provides another opportunity to identify the structures and avoid mistake. The advantages of this method also include: firstly, it is easy to learn and use, without requirements of special and expensive instruments like ICG method. Secondly, it could save time because the target bronchus is dissected and resected by utilizing the waiting period of intersegmental plane. Thirdly, because the bronchus is not resected at the moment of inflation, it is easy to achieve a full re-expansion with a relatively low pressure. This could reduce pressurerelated damage of lung caused by manual ventilation.

The bidirectional approach was also proposed by our team in 2020 (2). One of the main advantages is that it helps us to understand the anatomy of the hilar area from the posterior hilum, while it is not necessary to separate the S6 from the basal segments. It is less technically demanding, especially suitable for beginners. In PL approach alone, because we cannot get a whole picture of all branches of the inferior pulmonary vein, the identification of target vein is difficult. By the bidirectional approach, however, this problem could be resolved. Since the intersegmental plane is already visualized at this moment, the vein that run into the inflation area is to be cut; the vein that run into the deflation area is to be preserved. We do not even need to recognize each vein. The chest tube duration was relatively long in our group. It might be related to the two dissection areas made by "bidirectional" method. While this method is an advantage for understanding precise anatomy, it might be a disadvantage in chest tube duration. The dissection of interlobar fissure might be related to the prolonged air leakage.

\section{Conclusions}

The S10 segmentectomy is considered a difficult procedure among all segmentectomies. Although different methods were introduced, this is the first time in literature that we combine the pulmonary circulation single-blocking method and "bidirectional" method together for S10 segmentectomy. The preliminary result shows that it might be a useful method for S10 segmentectomy. It is safe with acceptable complications.

\section{Acknowledgments}

Funding: None.

\section{Footnote}

Reporting Checklist: The authors have completed the CARE reporting checklist. Available at https://shc.amegroups.com/ article/view/10.21037/shc-21-18/rc

Conflicts of Interest: All authors have completed the ICMJE uniform disclosure form (available at https://shc.amegroups. com/article/view/10.21037/shc-21-18/coif). The authors have no conflicts of interest to declare.

Ethical Statement: The authors are accountable for all aspects of the work in ensuring that questions related to the accuracy or integrity of any part of the work are appropriately investigated and resolved. All procedures performed in studies involving human participants were in accordance with the ethical standards of the institutional and/or national research committee(s) and with the Helsinki Declaration (as revised in 2013). Written informed consent was obtained from the patients for publication of this case report and any accompanying images. A copy of the written consent is available for review by the editorial office of this journal.

Open Access Statement: This is an Open Access article distributed in accordance with the Creative Commons Attribution-NonCommercial-NoDerivs 4.0 International License (CC BY-NC-ND 4.0), which permits the noncommercial replication and distribution of the article with the strict proviso that no changes or edits are made and the original work is properly cited (including links to both the formal publication through the relevant DOI and the license). See: https://creativecommons.org/ licenses/by-nc-nd/4.0/.

\section{References}

1. Zhang M, Wu QC, Ge MJ. Thoracoscopic electrocauterybased bidirectional S9-10 segmentectomy. Transl Cancer Res 2020;9:1303-6.

2. Zhang M, Mao N, Wu QC, et al. A novel method for distinguishing the intersegmental plane: pulmonary circulation single-blocking. J Thorac Dis 2021;13:362-5.

3. Sato M, Murayama T, Nakajima J. Thoracoscopic staplerbased "bidirectional" segmentectomy for posterior basal segment (S10) and its variants. J Thorac Dis 
2018;10:S1179-86.

4. Iwata H, Shirahashi K, Mizuno Y, et al. Surgical technique of lung segmental resection with two

doi: $10.21037 /$ shc-21-18

Cite this article as: Zhang M, Fu G, Chen X, Ge MJ.

Thoracoscopic bidirectional S10 segmentectomy: a case report.

Shanghai Chest 2022;6:6. intersegmental planes. Interact Cardiovasc Thorac Surg 2013;16:423-5. 